\title{
A GaAs MMIC-based X-band Dual Channel Microwave Phase Detector based on MEMS Microwave Power Sensors
}

\author{
Di Hua, Junyan Tan and Chunhua Cai \\ HoHai Univerity College of Internet of Things Engineering, Changzhou, Jiangsu 213022 China
}

\begin{abstract}
By sensing the output powers of two channels with MEMS microwave power sensors, a wideband 8$12 \mathrm{GHz}$ dual channel microwave phase detector is presented. In order to detect phase difference between reference signal and testing signal in entire $0-360^{\circ}$ at X-band, artificial $45^{\circ}$ phase lead and $45^{\circ}$ phase lag are introduced in two channels, additionally. The proposed phase detector is composed of power dividers, power combiners, $45^{\circ}$ (at $10 \mathrm{GHz}$ ) coplanar waveguide (CPW) transmission delay lines, MEMS capacitive power sensors and thermoelectric power sensors. The two CPW transmission lines result in $45^{\circ}$ phase lead and $45^{\circ}$ phase lag at $10 \mathrm{GHz}$, and the two different kinds of power sensors are used to detect the combined powers. The fabrication of the phase detector is compatible with GaAs Microwave Monolithic Integrated Circuit (MMIC) technology. The phase detection measurement is accomplished at $10 \mathrm{GHz}$ with $0-180^{\circ}$ phase shift between testing signals generated by a tunable analog phase shifter. Phase detection results in two channels show that the normalization of the measured results fit the calculated results well, though the results of phase lead have a little of deviation.
\end{abstract}

\section{Introduction}

Phase detectors are widely used in phase demodulators, phase-locked loops, and phase-measuring equipment. In these applications, phase detection is usually performed at relatively low frequencies, and frequently after down conversion of the original high-frequency signal [1]. Phase detection directly performed at microwave frequencies would, in many cases, lead to a reduction in hardware complexity. The existing technologies for microwave phase detection are based on diodes, CMOS and vector combination. Diode phase detectors take use of the square-law detection characteristic of diodes, but have problems with impedance matching [1-4]. CMOS phase detectors employ FET multiplier to realize phase detection, and will consume extra power, since DC bias is needed [5]. Meanwhile, diode phase detectors and CMOS phase detectors are sensitive to temperature variations. Based on vector combination and microwave power meters, vector combination phase detectors cannot detect phase difference between $-180^{\circ}$ to $+180^{\circ}$, since the output is symmetrical in $-180^{\circ}$ to $+180^{\circ}$ [6]. Also, the phase detector in [6] had not been realized on one chip.

Composed of a power combiner and a thermoelectric power sensor, the single channel microwave phase detector had been reported [7, 8]. The proposed phase detector has simple structure and does not consume any DC power, but the measured result shows that phase shift cannot be measured or deduced in 0 to $360^{\circ}$. In this paper, the dual channel microwave phase detector divides reference signal and testing signal into two channels, and introduces additional $45^{\circ}$ phase lead and $45^{\circ}$ phase lag between the reference signal and the testing signal ${ }^{\circ}$. The phase difference can be finally calculated through the outputs of the two kinds of power sensors. In channel I, the divided testing signal and the divided reference signal with additional $45^{\circ}$ phase delay are combined. The divided testing signal with additional phase delay is combined with the divided reference signal in channel II. The output powers of the two channels are detected by the MEMS power sensor. Two kinds of microwave power sensors are adopted because of the high powerhandling capability of the capacitive power sensor and the high sensitivity of the thermoelectric power [9-12]. The membrane of the MEMS capacitive power sensor will be slightly pulled down with low power applied. The power will be absorbed by two load resistors, and be converted to the thermo-voltage by the thermopiles. When the output powers are incidentally large, the output powers result a detectable displacement of the MEMS membrane. The movement can be measured capacitively. Finally, the phase difference between testing signal and reference signal can be deduced according to the detected output power of the two channels.

\section{Analysis and Design}

The proposed dual channel can be divided into two parts. Dual channel structure is a four-port signal processing structure, and outputs the signals containing the phase difference between the reference signal and the testing 
signal in two channels. The other parts are microwave power sensors, including MEMS thermoelectric power sensors and MEMS capacitive power sensors.

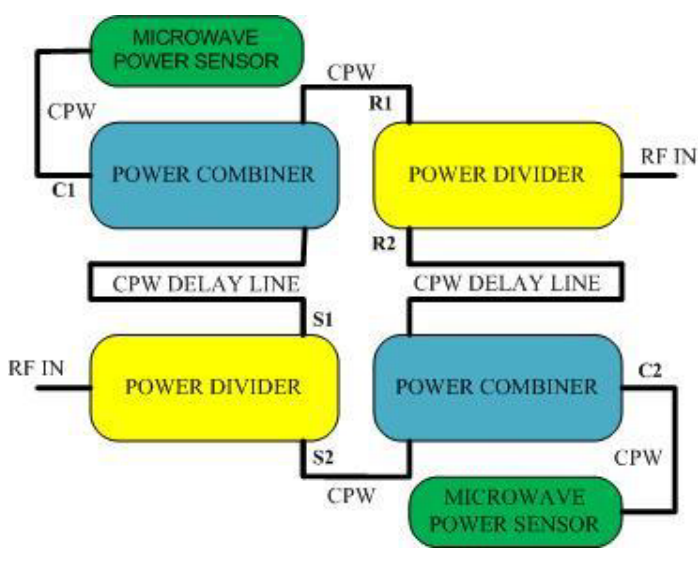

Figure 1. Schematic overview of the dual channel phase detector

\subsection{Dual Channel Structure}

The dual channel structure is composed of two power dividers), two power combiners and two phase shifters. Fig. 1 shows the schematic circuit of the dual channel microwave phase detector. Testing signal Us and reference signal Ur are applied to input ports, and the two signals should have the same power in order to simplify the calculation. Then, testing signal is divided into Us1 and Us2 by one power divider, which have the same magnitudes and the phase shifts, and reference signal is divided into Ur1 and Ur2 by the other power divider, respectively. Ur1 transmits through a $45^{\circ}$ phase shifter, and be combined with Us1 by one power combiner. Symmetrically, Ur2 transmits through another $45^{\circ}$ phase shifter, and be combined with Us2 by the other power combiner.

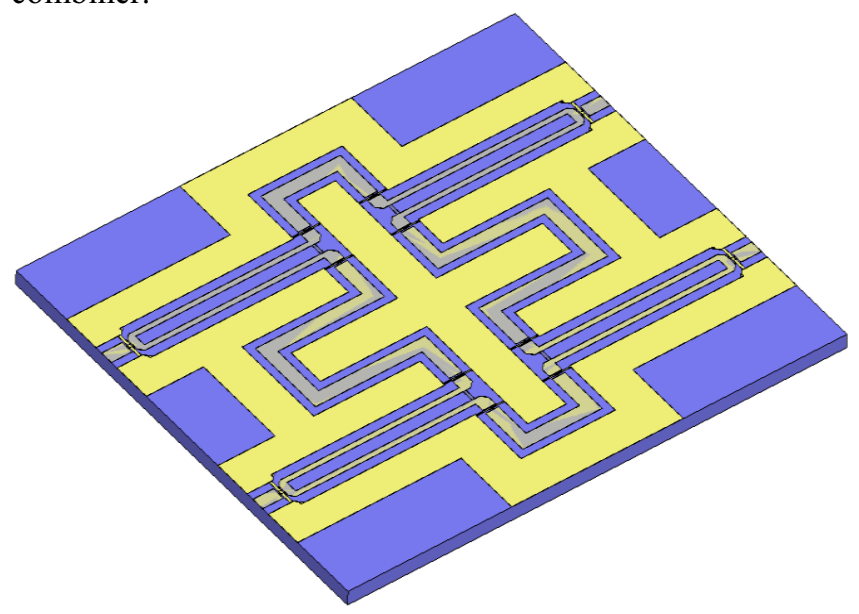

(a)

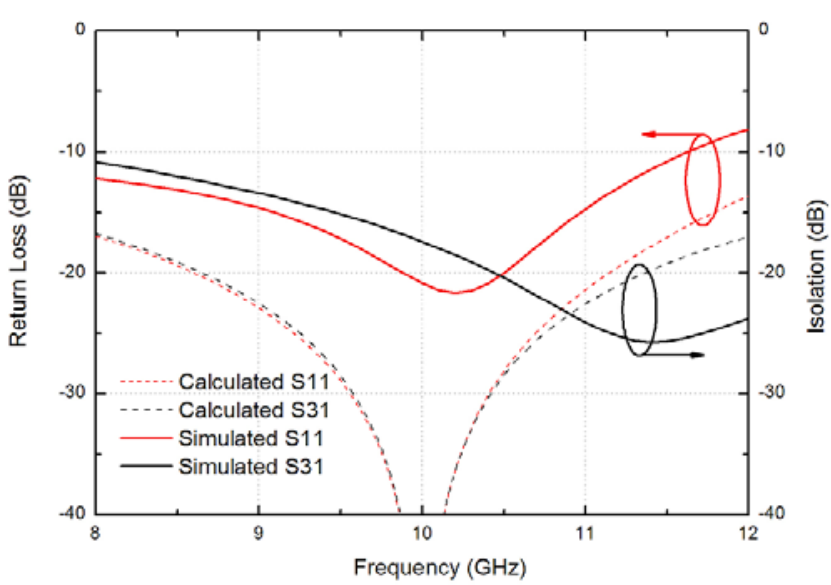

(b)

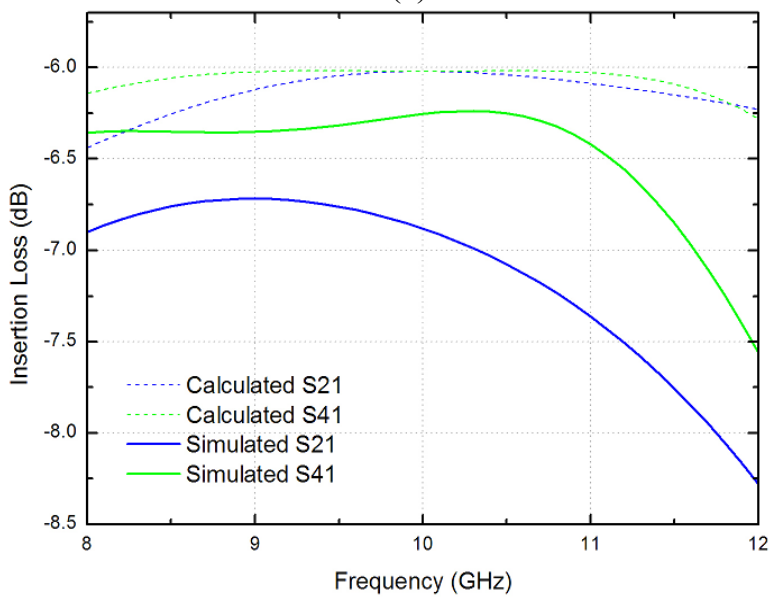

(c)

Figure 2. (a) The simulating dual channel structure and (b) (c) its simulated S-parameters

Fig. 2(a) shows the dual channel structure. As can be seen in Fig. 2, over a $75 \%$ bandwidth centered at $10 \mathrm{GHz}$, the simulated return loss of input ports is less than $13.15 \mathrm{~dB}$ and the insertion losses between port 1,2 and 1, 4 are $-7.75 \mathrm{~dB}$ and $-6.85 \mathrm{~dB}$, respectively. The isolation between input ports 1,3 is less than $-12.02 \mathrm{~dB}$. Since the insertion loss of ports 1,2 and ports 1, 4 are almost equal, the insertion loss $\mathrm{S} 12$ and $\mathrm{S} 14$ are defined to $\mathrm{S}$ in order to simplify the research. When the testing signal and reference signal are applied to Ports 1, 3, the out put powers of Ports 2, 4 can be expressed as

$$
\begin{aligned}
& P_{\text {out } 2}=Z_{0} S^{2} U^{2}\left(1+\cos \left(\varphi+45^{\circ}\right)\right) \\
& P_{\text {out } 4}=Z_{0} S^{2} U^{2}\left(1+\cos \left(\varphi-45^{\circ}\right)\right)
\end{aligned}
$$

\subsection{MEMS capacitive power sensor and thermoelectric power sensor}

The output powers of the dual channel structure are detected by two kinds of microwave power sensors, the MEMS capacitive power sensor and the thermo-electric power sensor. The two kinds of power sensors are adopted to increase the detective range. When relative high level signal is applied, the membrane will be attracted. While relative low level signal is inputted, the signal will be detected by the thermo-electric power sensor. 


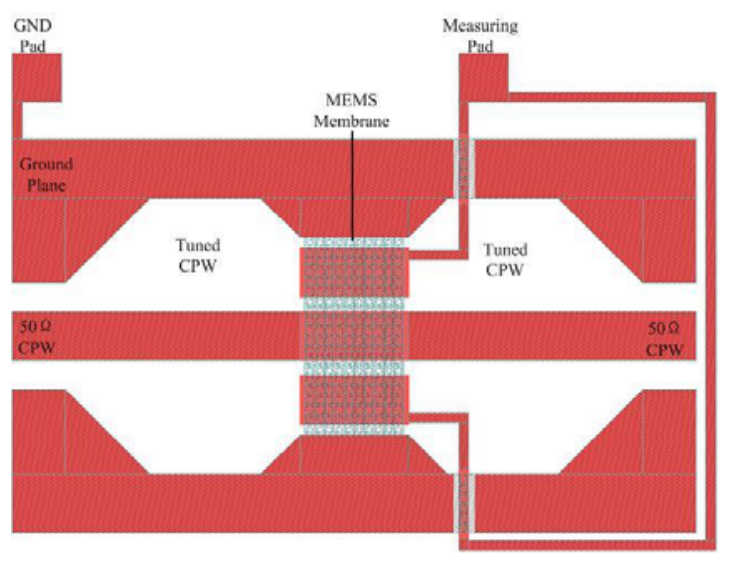

(a)

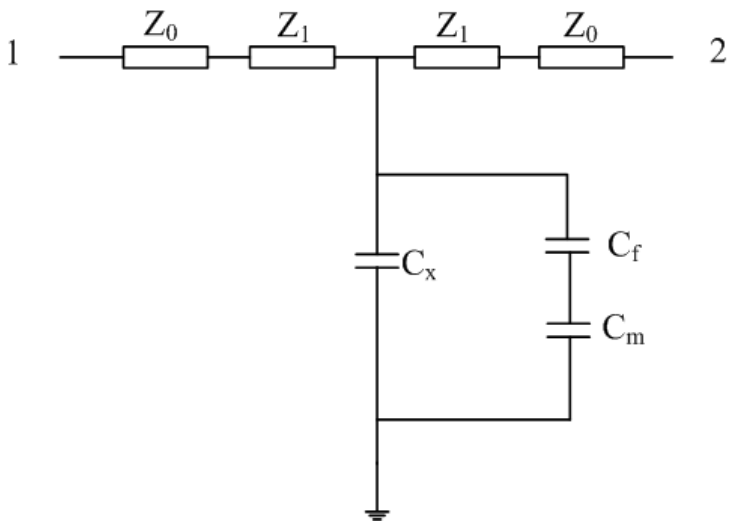

(b)

Figure 3. (a)Schematic view of the MEMS capacitive power sensor (b) the corresponding circuit

The MEMS capacitive power sensor is composed of $50 \Omega \mathrm{CPW}$, tuned CPW and a fixed-fixed MEMS membrane suspended on the signal line in Fig. 3(a). The anchors of the membrane are connected to the ground plane of the CPW, while the CPW is terminated by two parallel $100 \Omega$ TaN resistors. Due to the discontinuity of the shunt MEMS membrane, the CPW near the membrane are tuned to realize well impedance matching. Fig. 3 (b) shows the schematic view and the schematic circuit of the capacitive power sensor, where Z1 is the characteristic impedance of the tuned $\mathrm{CPW}, \mathrm{Cx}$ is the capacitance between the center signal line and the membrane, $\mathrm{Cf}$ is the fringing capacitance between the center signal line and the measuring pad, $\mathrm{Cm}$ is the capacitance between the measuring pad and the membrane. Since $\mathrm{Cf}$ is relatively small, $\mathrm{Cf}$ and $\mathrm{Cm}$ can be removed for simplification.

When the processed microwave signals are applied to the two capacitive power sensors, the membrane is attracted. The thermoelectric power sensor is composed of $50 \Omega$ terminating resistor and thermo-couples, whose principle is based on Seebeck effect. The thermo-couple is two jointed different materials. When relative low level combined signals are applied to the MEMS capacitive power sensor, the signals will not attract the membrane. Almost all the combined signals are absorbed by the terminating resistors and converted to DC thermovoltage output by the thermopiles [13].

\section{Fabrication}

The fabrication of the microwave phase detector is compatible with GaAs MMIC process. Surface micromachining technology is used to fabricate the thermopiles, and bulk micromachining technology is employed to reduce thermal loss and increase the sensitivity of the power sensor. In this detector, the CPW is designed to have $50 \Omega$ characteristic impedance. In [14], the process steps of the detector are briefly described as follows

(1) The thermopiles are made of $\mathrm{Au}$ and $\mathrm{n}+\mathrm{GaAs}$ with a doping concentration of $1.0 \times 1018 \mathrm{~cm}-3$. The $\mathrm{Au}$ is made by sputtering a $500 / 2000 \AA \mathrm{AuGeNi} / \mathrm{Au}$ layer and using a lift-off process, and the $\mathrm{n}+\mathrm{GaAs}$ is made of an ion implantation layer. The thermopile has length of $80 \mu \mathrm{m}$.

(2) A TaN layer is sputtered and patterned to form the load resistors and isolation resistors with the square resistance of 25 .

(3) The lower plates of the MIM capacitors are made by evaporating a $0.3-\mu \mathrm{m}$-thick $\mathrm{Au}$ layer, and $\mathrm{AuGeNi} / \mathrm{Au}$ layer is used as adhesion layer.

(4) A $1000 \AA \mathrm{Si} 3 \mathrm{~N} 4$ is deposited to form the dielectric layers of the MIM capacitors by PECVD.

(5) A $500 / 1500 / 300 \AA \mathrm{Ti} / \mathrm{Au} / \mathrm{Ti}$ seed layer is evaporated and patterned. After removing the top Ti layer, the CPW transmission line and the upper plates of the MIM capacitors are formed through electroplating a 2$\mu \mathrm{m}$-thick Au layer.

(6) The substrate is thinned to about $100 \mu \mathrm{m}$ by a wafer grinding process, and the substrate underneath the thermopiles and the load resistors is etched to $20 \mu \mathrm{m}$ in order to reduce thermal losses using dry etching technology.

(7) The sacrificial layer of polyimide below the membrane and the air bridge is removed using a developer and the alcohol is utilized to get rid of the residual water in the MEMS membrane.

The fabrication of the microwave phase detector is compatible with GaAs MMIC process. Surface micromachining technology is used to make the thermopiles, and bulk micromachining technology is employed to reduce thermal loss and increase the sensitivity of the power sensor in Fig. 4. In this detector, the $\mathrm{CPW}$ is designed to have $50 \Omega$ characteristic impedance. In [8], the process steps of the detector are briefly reported. 


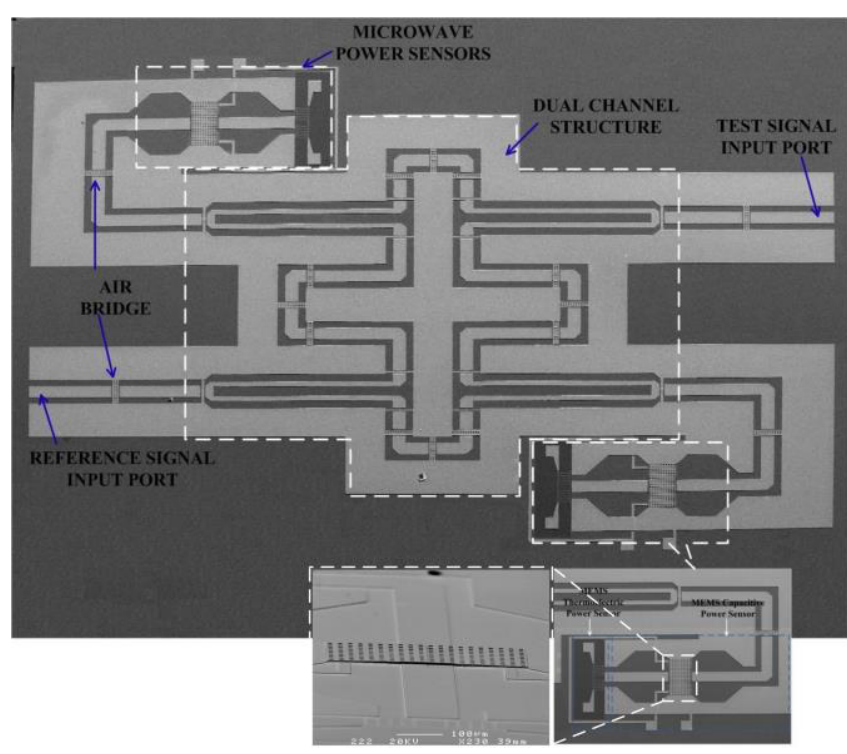

Figure 4. SEM picture of the whole dual channel microwave phase detectora and the MEMS membrane

\section{Measurement Result}

Phase detection environment is described as follow. The testing signal and the reference signal are generated by an Agilent power splitter and an analog phase shifter, which can make the phase shift change between 0 to $240^{\circ}$. A nut is used to control the phase delay of the phase shifter. The initial phase delay is about $180^{\circ}$ instead of 0 because of the phase delay of the adapters and the connecting cables. According to the instruction book of the phase shifter, the phase delay step is about $9^{\circ}$ at $10 \mathrm{GHz}$ per round. The MEMS capacitive power sensor has the advantage of high power-handling capability, while its disadvantage is that relatively low test signal and reference signal can't attract the membrane. Since the phase detection environment is restricted by the maximum output power of the signal generator, the phase detection measurement is accomplished by the thermoelectric power sensor only, while the MEMS capacitive power sensor is measured, separately.

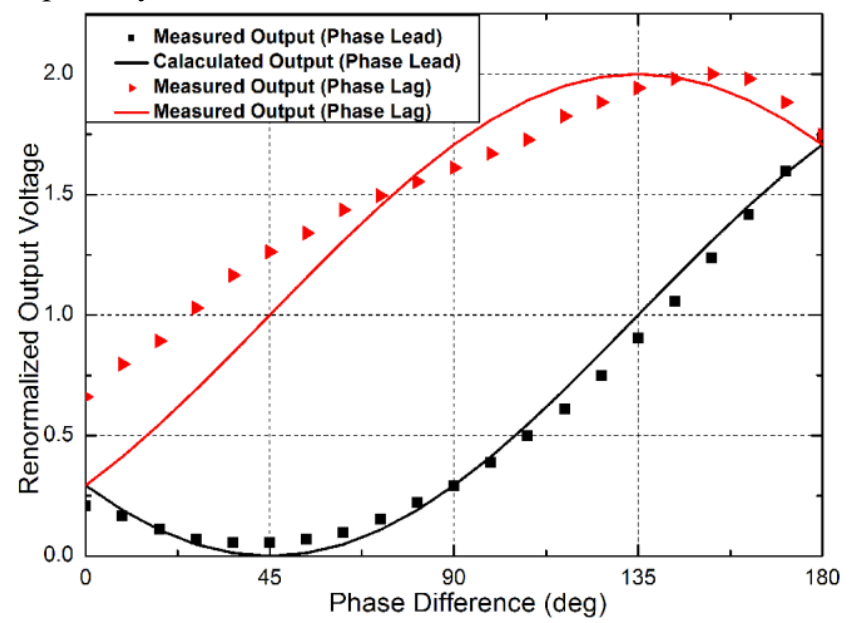

(a)

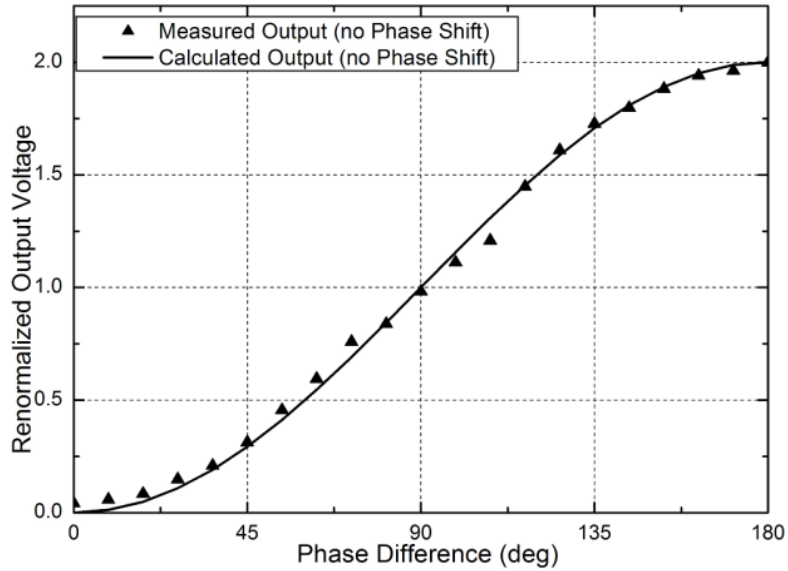

(b)

Figure 5. Normalizations of measured results and calculated results with $45^{\circ}$ phase lead, $45^{\circ}$ phase lag and no phase shift

Fig. 5 shows the normalized phase detection results in three different situations. Fig. 5 (a) show additional $45^{\circ}$ phase lead and $45^{\circ}$ phase lag between testing signal and reference signal, respectively, and Fig. 5 (b) shows no phase shift is added. As can be seen in Fig. 5, the measured results and the calculated results are matched well, which means that the phase detection principle is validated, while the results in Fig. 5 (a) have a little of deviation. The reason of deviation may be that the discreteness of the fabrication process makes the detector asymmetrical.

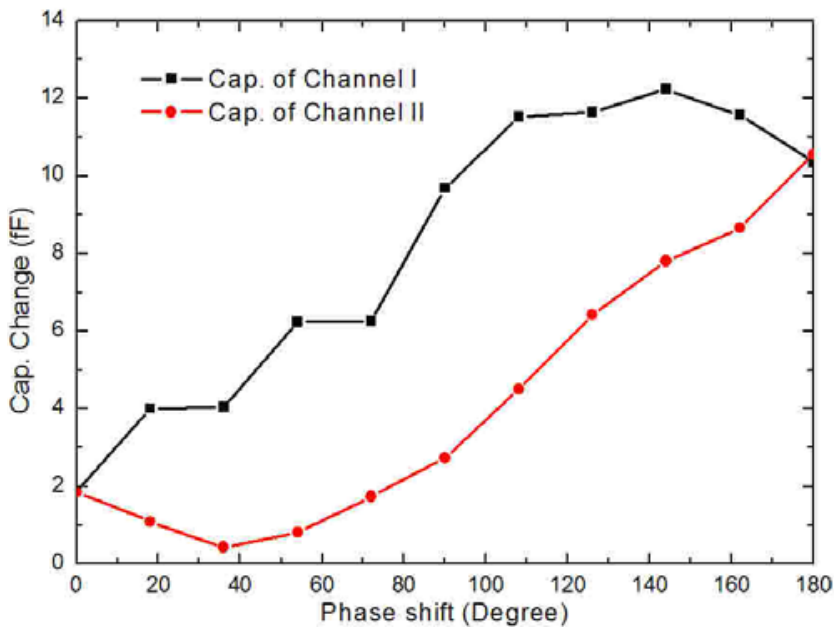

Figure 6. Measured capacitance change versus phase shift with $25 \mathrm{dBm}$ signal applied

Fig.6 shows the measured capacitance change versus the phase shift in two channels. Two methods are involved to make the capacitance change can be detected: 1. the maximum power level of the signal generator are applied. 2. the insertion loss of the measurement environment is optimized to reduce the power loss. The calculated phase differences from Fig. 5 and 6 include the effective phase difference and the initial phase difference. The simulation result of the dual channel structure shows that the insertion loss of channel I and II are $6.4 \mathrm{~dB}$ 
and7.0dB, respectively. The maximum power levels of the testing signal and the reference signal should be less than $20 \mathrm{dBm}$, which means that the power level of the signal generator should be less than $23 \mathrm{dBm}(3 \mathrm{~dB}$ insertion loss of the power divider, used to generate the test and reference signals). For the thermoelectric power sensor, the high power limitation of the input testing signal and reference signal is estimated to be $20 \mathrm{dBm}$.

The dimension of the MEMS membrane is $400 \mu \mathrm{m} * 200 \mu \mathrm{m} * 2 \mu \mathrm{m}$ with $20 \mu \mathrm{m} * 10 \mu \mathrm{m}$ holes formed. The goal of the small holes is to release the sacrificial layer under the membrane. The designed height of the initial air gap is $1.6 \mu \mathrm{m}$. A pair of $100 \mu \mathrm{m} * 200 \mu \mathrm{m} * 0.3 \mu \mathrm{m}$ measuring electrodes are fabricated between the anchors and the center signal line with a $0.1 \mu \mathrm{m}$ thick $\mathrm{Si}_{3} \mathrm{~N}_{4}$ layer deposited. The calculated initial capacitance between the electrodes and the membrane is $0.110 \mathrm{pF}$, and the fringing capacitance is neglected. Figure 7 shows the simulated displacement and the corresponding capacitance versus different voltages using ConventorWare v2006. The capacitive microwave power sensor is measured at 8,9 , 10,11 , and $12 \mathrm{GHz}$, and the average sensitivity is $7.2 \mathrm{fF}$ $\mathrm{W}^{-1}[15]$.

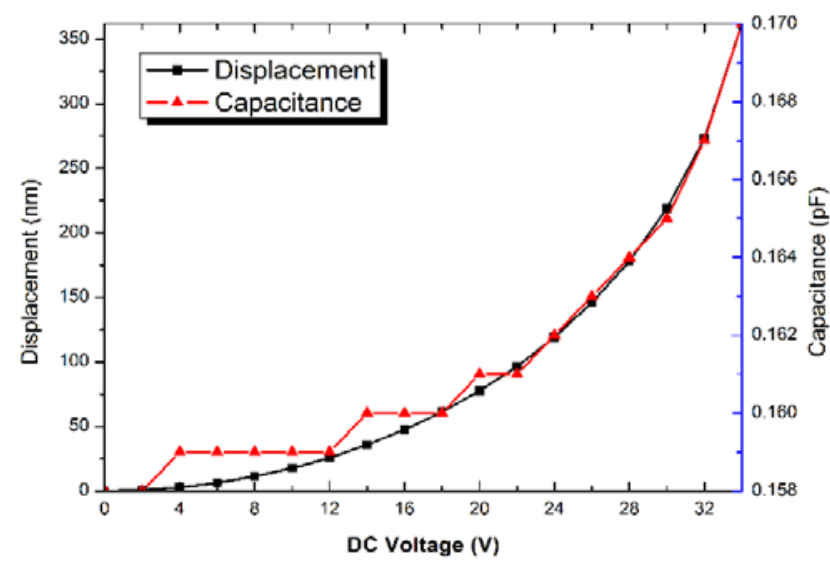

Figure 7 Simulated displacement and the corresponding capacitance versus different voltages

\section{Conclusion}

In order to extend the detectable phase range, a wideband $8-12 \mathrm{GHz}$ symmetrical dual channel microwave phase detector is presented in this paper, and the phase detector is accomplished with GaAs MMIC technology. The detector takes use of $45^{\circ}$ phase lead and $45^{\circ}$ phase lag in two channels, and two thermoelectric microwave power sensors are used to detect the two output powers. The MEMS capacitive power sensor provides wide powerhandling capability, which means that the proposed phase detector has higher power-handling capability than diodebased phase detectors. Phase shift between reference signal and testing signal can be calculated, determinately and uniquely. The measured S-parameters show that the detector are well impedance matched, and the normalizations of measured output voltages agree well with the calculated results in different situations. The dual channel microwave phase detector can be widely applied to phase-measuring equipment and radar systems.

\section{Acknowledgement}

This work is supported by the Fundamental Research Funds for the Central Universities (2014B01914).

\section{References}

1. Ohm G and Alberty M. "Microwave Phase Detectors for PSK Demodulators," IEEE Microwave Theory and Techniques 1981, 29(7), 724-731.

2. Mohammad M. "A Wide Dynamic-range Phase Detector for Advanced Communication Systems Application," WESCANEX 93. 'Communications, Computers and Power in the Modern Environment.' IEEE Conference Proceedings, May, 1993, pp. 192-195.

3. Setc B. and Reckko C. "Broadband Microwave Phase Detector with Diode Ring," IEEE Microwaves, Radar and Wireless Communications, MIKON-2000, 13th International Conference, May, 2000, 1, pp. 65-68

4. Slomski R and Stec B. "Broadband microwave phase discriminator with ring detectors," IEEE Microwaves, Radar and Wireless Communications, MIKON-2004, 15th International Conference, May, 2004, 3, pp: 936-939.

5. Shahriary I, Lin S.T. and Weller K. "A Practical Wide Band GaAs Phase Detector," Microwave and Millimeter-Wave Monolithic Circuits 1982, 82(1): 47-49

6. Lehto $\mathrm{A}$, Tuovinen $\mathrm{J}$, Borić $\mathrm{O}$ and Räisänen A. "Accurate Millimeter Wave Antenna Phase Pattern Measurements Using the Differential Phase Methode with Three Power Meters," IEEE Antennas and Propagation, 1992, 40(7), 851-853.

7. D. Hua, X.P. Liao and Y.C. Jiao, "A MEMS phase detector at Xband based on MMIC technology," in Sensors, 2009 IEEE, 2009, pp. 506-508.

8. D. Hua, X.P. Liao and Y.C. Jiao, "X-band microwave phase detector manufactured using GaAs micromachining technologies," Journal of Micromechanics and Microengineering, 2011, 21(3): 035019

9. Y. Cui, X.P. Liao and Z. Zhu, "A novel microwave power sensor using MEMS fixed-fixed beam," in Sensors, 2011 IEEE, 2011, pp. 1305-1308.

10. Z.X. Yi, X.P. Liao, Z. Zhu; , "An $8-12 \mathrm{GHz}$ capacitive power sensor based on MEMS cantilever beam," Sensors, 2011 IEEE , vol., no., pp.1958-1961, 28-31 Oct. 2011

11. D.B. Wang, X.P. Liao, T. Liu, "A Novel Thermoelectric and Capacitive Power Sensor With Improved Dynamic Range Based on GaAs MMIC Technology," Electron Device Letters, IEEE , vol.33, no.2, pp.269-271, Feb. 2012

12. De-Bo Wang, Xiao-ping Liao, "A terminating-type MEMS microwave power sensor and its amplification system," J. Micromech. Microeng. 2010, 20(7):075021

13. Milanovic V, Hopcroft M, Zincke C.A., et al. "Optimization of CMOS MEMS microwave power sensors," Circuits and Systems, Proc. IEEE ISCAS '99, May, 1999, 5:144 -147.

14. D. Hua, X.P. Liao and J.Q. Huang, "A GaAs MMIC-based dual channel microwave phase detector at X-band," in Sensors, 2012 IEEE, 2012, pp. 1-4.

15. Y. Cui, X.P. Liao and Z. Zhu, "A novel microwave power sensor using MEMS fixed-fixed beam," in Sensors, 2011 IEEE, 2011, pp. 1305-1308. 